\title{
APLICACIÓN DE LA TERAPIA DE INTERACCIÓN PADRES-HIJOS EN UN CASO DE TRASTORNO NEGATIVISTA DESAFIANTE
}

\author{
RAFAEL FERRO ${ }^{1}$, CARMEN VIVES ${ }^{2}$ Y LOURDES ASCANIO $^{1}$ \\ ${ }^{1}$ Centro de Psicología Clínica CEDI, Granada \\ ${ }^{2}$ Facultad de Psicología, Universidad de Granada
}

\begin{abstract}
Resumen: La terapia de interacción padres-hijos (PCIT) es una forma de terapia para tratar problemas de la infancia que integra la terapia de juego tradicional y los métodos clásicos operantes. Ha demostrado ser útil en problemas como: desobediencia, hiperactividad, agresividad, mentiras, problemas de conducta en clase, robos, conductas auto-lesivas, rabietas, etc. El Trastorno negativista desafiante (TND) se caracteriza por una actitud de oposición hacia la autoridad, incluyendo berrinches, gritos, pataletas, discusiones de las reglas y las peticiones, etc. En este trabajo se describe la aplicación de PCIT en una niña de 3 años con TND. El tratamiento duró 11 meses consiguiéndose resultados positivos sobre las conductas problema de la niña y su mantenimiento durante un seguimiento de un año.
\end{abstract}

Palabras clave: Terapia de interacción padre-hijo, Trastorno negativista desafiante, tratamiento, seguimiento

Implementation of parent-child interaction therapy in a case of oppositional defiant disorder

\begin{abstract}
The parent-child interaction therapy (PCIT) is a type of therapy for treating childhood problems. It integrates traditional play therapy and classical operant methods. It has proved to be useful in problems such as disobedience, hyperactivity, aggression, lying, classroom behavior problems, stealing, self-injurious behaviors, tantrums, amongst others. Oppositional Defiant Disorder (ODD) is characterized by an oppositional attitude toward authority, including temper tantrums, screaming, tantrums, arguments as to rules and requests, and so forth. This paper describes the application of PCIT to a girl of 3 years with ODD. Treatment lasted 11 months, providing positive results with problem behaviours, maintained through the one year follow up.
\end{abstract}

Keywords: Parent-child interaction therapy, oppositional defiant disorder, treatment, follow up.

\section{INTRODUCCIÓN}

La terapia de interacción padres-hijos ( $p a-$ rent-child interaction therapy, PCIT; Eyberg, 1988, 1999; Hembree-Kigin y McNeil, 1995) es una terapia breve para tratar problemas de la infancia, y ha sido considerada como un tratamiento psicológico con apoyo empírico (Brink-

\footnotetext{
Recibido: 5-mayo-2010; aceptado: 2-julio-2010

Correspondencia: Rafael Ferro García, Centro de Psicología Clínica CEDI, Avda. Constitución 25, $7^{\circ}$ Izqda. 18014 Granada, España.

Correo-e: rferro@cop.es.

Web: http://www.cop.es/colegiados/GR00777
}

meyer y Eyberg, 2003). Surge a final de los años 80 e integra la terapia de juego tradicional y los métodos operantes. Ha demostrado ser útil en una serie de problemas infantiles como: desobediencia, hiperactividad, agresividad y conductas destructivas, mentiras, problemas de conducta en clase, robos, conductas auto-lesivas, rabietas, retraso en el desarrollo, ansiedad generalizada, etc. (Hembree-Kigin y McNeil, 1995).

Se trabaja con las interacciones entre los padres y el hijo a través del juego, y tiene dos fases. En la primera fase, denominada interacción directa infantil, el objetivo es establecer una relación afectuosa entre ellos. Aquí, el tratamiento se centra en enseñar a los padres a usar 
la atención selectiva, incrementando las conductas pro-sociales con la atención de los padres mientras que las conductas indeseables son reducidas, ignorándolas. La segunda fase se denomina interacción dirigida hacia los padres, y está dedicada a estrategias de disciplina. Se les enseña a los padres cómo dar consecuencias consistentes a las conductas de sus hijos. El tratamiento se aplica entre 8 y 12 sesiones, con sesiones extras si son necesarias, y un seguimiento a 1, 3, 6 y 12 meses. Tiene 7 pasos: En el paso 1 , se realiza la evaluación pre-tratamiento del funcionamiento familiar y del feedback de la familia con el niño (conlleva un periodo de 1 ó 2 sesiones). El paso 2, consiste en enseñar habilidades de terapia de juego conductual (en 1 sesión) a través de modelos de los terapeutas. Durante el paso 3 se entrena a la propia familia las habilidades de terapia de juego conductual (de 2 a 4 sesiones), aplicándolas durante las sesiones clínicas. En el paso 4, se enseñan técnicas de disciplina (en 1 sesión), presentando de nuevo modelos el terapeuta. Es en el paso 5 donde se realiza el entrenamiento de técnicas de disciplina con la familia (de 4 a 6 sesiones). En el paso 6, se hace una evaluación post-tratamiento del funcionamiento y del feedback de la familia y el niño (aproximadamente, 1 ó 2 sesiones). Finalmente el paso 7 , consiste en realizar sesiones extraordinarias si son necesarias y el seguimiento (Ferro, Vives y Ascanio, 2009). Como proponen McNeil, Filcheck, Greco, Ware y Bernard (2001), el orden de las fases y los pasos en su aplicación se adaptan a cada caso. Se realizan grabaciones en video para permitir un mejor análisis del funcionamiento familiar y para demostrar a las familias los errores y los aciertos en el mismo.

Hay muchos estudios sobre su efectividad $\mathrm{y}$ eficacia, en los que se han demostrado mejoras clínicas y estadísticamente significativas con niños con conductas problema, así como la generalización de los resultados en la casa (Hembree-Kigin y McNeil, 1995; Herschell, Calzada, Eyberg, y McNeil, 2002), frente a listas de espera o grupos de control (Bagner y Eyberg, 2007; Chaffin, Silovsky, Funderburk, Valle, Brestan, Balachova, Jackson, Lensgraf, y Bonner, 2004; Eyberg y Matarazzo, 1980; McNeil, Capage, Bahl y Blanc,
1999; Schuhmann, Foote, Eyberg, Boggs y Algina, 1998), y manteniendo los resultados durante varios años en el seguimiento (Eyberg, Funderburk, Hembree-Kigin, McNeil, Querido y Hood, 2001; Hood y Eyberg, 2003).

El trastorno negativista desafiante (TND) se caracteriza por una actitud de oposición manifiesta hacia toda figura de autoridad (padres y maestros). Abarca un patrón de conductas que implica discusiones continuas, desafiar las reglas y las peticiones, oponerse a las figuras de autoridad y berrinches. Puede incluir otros problemas como molestar a otras personas deliberadamente, enojarse con facilidad, el uso de palabrotas y culpar a otros por las faltas propias. Entre los 3 y 7 años los comportamientos del TND se vuelven tan problemáticos que llegan a interferir en el funcionamiento diario de la familia (Connor, 2002). Se estima que afecta entre el 2 y el $16 \%$ de la población infantil y que esta variabilidad en los datos depende de varios factores: los criterios diagnósticos empleados para el estudio, los métodos de evaluación, la fuente de información y el tipo de muestra (Burke, Loeber, Birmaher, 2002; Cardo, Meisel, Garcia-Banda, Palmer, Riutort, Bernad, y Servera, 2009).

Para Barkley, Edwards y Robin (1999) hay cuatro factores cuya combinación explica la aparición de la conducta negativista y desafiante de niños y adolescentes: las prácticas de crianza, las características del niño o adolescente, las características de los padres y algunos factores contextuales. Entre las características del niño, se ha relacionado la personalidad con este tipo de problemática (Carrasco y del Barrio, 2007). En cuanto a las prácticas de crianza, la calidad de las relaciones padres-niño está asociada con la gravedad de la desobediencia, el desafío y las pautas de conducta agresiva, y también con la persistencia de esas conductas a lo largo del desarrollo. La consistencia y la inconsistencia en la educación de los padres está vinculada con la presencia de conductas agresivas (Rodríguez, Del Barrio y Carrasco, 2009). Es decir, estos padres suelen proporcionar poca atención o poco reforzamiento a las conductas pro-sociales o adecuadas del niño. Debido a esto, los niños experimentan éxito periódico en evitar las demandas reforzándose 
así la conducta coercitiva y negativista y poniendo la base del desafío y de los problemas de conducta en el futuro adolescente.

No cabe duda que los trastornos de conducta son un problema en aumento en la sociedad actual. Cada día se presentan más casos, en el entorno familiar, social y escolar, de desobediencia extrema, de conducta desafiante y de comportamientos disociales (Portugal y Arauxo, 2004). A continuación se expone un caso de TND de una niña de tres años y su evolución a lo largo del tratamiento con la PCIT.

\section{MÉTODO}

\section{Participante}

Elena tenía tres años cuando sus padres acudieron a consulta. Era la mayor de dos hermanos y acababa de finalizar el $2^{\circ}$ curso de preescolar. Presentaba conductas negativistas y desafiantes, desobediencia, rabietas continuas de alta frecuencia e intensidad, acompañadas de autolesiones, patadas, bofetadas, insultos, y en ocasiones se orinaba encima. Esto ocurría con sus padres y sus abuelos. Tenía una dependencia excesiva con la madre, demandando constantemente su atención y rechazando al resto de adultos, incluido el padre.

Mostraba algunos problemas a nivel social, no saludaba y no se relacionaba con otros niños. En cuanto a su lenguaje no era acorde con su edad, hablaba con poca frecuencia y con monosílabos y su topografía vocal era deficiente. Tenía temores nocturnos desde hacía tres meses, llegándose a despertar tres y cuatro veces en una misma noche.

\section{Análisis del caso}

El análisis del caso y su evaluación se realizó a través de una entrevista semiestructurada elaborada en el propio centro, y a través de la visualización de las grabaciones en video tanto de las conductas de la niña como de la interacción con los padres y terapeutas. Estas grabaciones se realizaron con el permiso expreso de sus padres. Elena cumplía los criterios diagnósticos del DSM-IV (APA, 1994) del trastorno negativista desafiante (F91.3). Es decir, un comportamiento negativista y desafiante (pataletas o rabietas, discusiones y desafíos a los adultos, no cumplir las demandas de éstos, etc.) que duraba más de 6 meses, provocando un deterioro clínicamente significativo en la actividad social y académica.

Se hipotetizó que las conductas estaban mantenidas por atención social de los padres y como conductas de evitación y/o escape de algunas tareas. Las rabietas tenían una frecuencia de 4 ó 5 al día con una duración aproximada de 30 minutos. Durante éstas, lloraba, agredía, se autolesionaba, etc. Los padres reconocieron utilizar diferentes consecuencias, dependiendo del momento, del tiempo disponible y de su estado de ánimo. Como por ejemplo: ponerla de pie, regañarle, le daban leche con un biberón, etc. Interpretaban que el problema de Elena derivaba de los celos hacia su hermano pequeño. Los padres eran asistemáticos e inconsistentes. Utilizaban amenazas y castigos y la tasa de reforzamiento positivo era muy baja. Tendían a interpretar como intencionadas las conductas problemas de la niña. Se definieron operacionalmente las conductas problema que presentaba:

1. Negativas: Decir «no» y/o mover la cabeza ante una orden.

2. Desobedecer. Ante una orden de algún adulto (terapeutas y/o padres) no seguir tal instrucción o realizar otra cosa diferente.

3. Escupir a un adulto. De forma espontánea la niña echaba por la boca un poco de saliva dirigida al adulto.

4. Agredir. Se consideraron todas aquellas conductas dirigidas al adulto o a un objeto con la funcionalidad de infringir daño.

5. Autolesiones: Todas aquellas conductas dirigidas a infringirse daño a ella misma.

6. Rabietas: Se consideraron tales conductas cuando la niña: lloraba, pataleaba, gritaba, etc.

También se consideró como una conducta objetivo de Elena: Obedecer. Como el seguimiento inmediato después de la primera orden formulada por el padre o terapeuta. 


\section{Intervención}

Se aplicó una versión adaptada del procedimiento propuesto en el manual (Hembree-Kigin y McNeil, 1995). El tratamiento duró 11 meses, con un total de 11 sesiones y un seguimiento de un año. Todas las sesiones fueron grabadas en video parcialmente, a excepción de la sesión 3 y la 5. Cada sesión tuvo una duración aproximada de una hora, dedicando la primera media hora a entrenar a los padres en las técnicas, a revisar y realizar los análisis funcionales y plantear dudas y situaciones problemáticas que habían surgido durante ese período de tiempo. En la segunda media hora se presentaban modelos por parte de los terapeutas y/o se moldeaba a los padres las conductas objetivo a través de una situación de juego. En esta parte de la sesión se grababa a la niña mientras interactuaba con los terapeutas y/o con sus padres.

Se les pidió a los padres que registraran todas las conductas problema de Elena y además, se les enseñó a realizar un análisis funcional descriptivo tipo A-B-C, en el que A representa los antecedentes de la conducta, B la conducta misma y $\mathrm{C}$ las consecuencias. Se le dio la posibilidad de contactar con los terapeutas a través de un correo electrónico o por teléfono, permitiendo de manera casi inmediata, corregir los errores, moldear y reforzar los objetivos.

Las dos fases de PCIT se aplicaron casi simultáneamente, ya que la paciente presentaba intensas y frecuentes rabietas y los padres no sabían cómo ponerlas bajo control. La primera fase de interacción directa infantil, fue el objetivo principal en las primeras sesiones. Como las rabietas tenían una frecuencia e intensidad muy alta y una forma peligrosa para la niña, se decidió aplicar el procedimiento de tiempo fuera, tal y como se explica en el manual. En la segunda fase de interacción dirigida hacia los padres, fueron entrenados a dar órdenes y aplicar de forma consistente consecuencias en función de la conducta de la niña.

Se les dieron por escrito unas instrucciones generales y unas instrucciones específicas de cómo dar una orden y conseguir su seguimiento. Estas últimas consistían en dar una orden, esperar su seguimiento y si ocurría, alabar el mismo. Si había desobediencia se exigía el se- guimiento de la orden repitiendo ésta y guiándola físicamente. También, se dijo a los padres que cuando aparecía una rabieta debían aplicar un tiempo fuera. A continuación, se describen las sesiones clínicas y la evolución del caso a través del tratamiento:

Sesión $1^{a}$ : Se recogieron los datos de la historia del caso y de las conductas disruptivas. Se realizó una grabación de 51 minutos de la interacción de los padres y la niña, se les dijo que intentaran jugar con ella. Durante el vídeo aparecieron negativas, desobediencia, y una rabieta de 37 minutos acompañada de agresiones. Después se les presentó a los padres modelos y se les instruyó sobre: cómo dar órdenes, cómo reforzar su seguimiento, cómo hacer correcciones, y cómo aplicar el tiempo fuera. Se les recomendó que en adelante, jugaran diariamente con Elena unos 15 minutos.

Sesión $2^{a}$ : Se corrigieron los fallos observados durante el vídeo de la sesión anterior. Los padres repetían muchas veces la misma orden, aplicaban el tiempo fuera antes de tiempo, prestaban atención a las pataletas y a las agresiones y no extinguían los insultos. En una situación de juego se presentaron modelos de la forma de interactuar. En esta sesión apareció una rabieta con el terapeuta que duró 2 minutos, en la que se aplicó un tiempo fuera. También surgieron negativas y desobediencias que fueron extinguidas, ignorándolas y consiguiendo su seguimiento.

Sesión $3^{a}$ : No hubo grabación, ya que en esta sesión apareció una rabieta de forma espontánea en sala de espera del centro, donde no se tenía instalado el equipo de grabación. La rabieta duró 33 minutos. La intensidad de ésta fue muy alta con llantos, gritos, autolesiones (se tiraba de las comisuras de la boca con las dos manos con fuerza, se tiraba del pelo, se frotaba los ojos con mucha fuerza), agresiones (daba patadas y manotazos cuando alguien se acercaba, se mordía el vestido). Durante toda la rabieta se aplicó de forma sistemática el tiempo fuera. Entre la $3^{a}$ y la $4^{\mathrm{a}}$ sesión, la terapeuta se puso en contacto con la madre por teléfono. Ella comentó una rabieta de alta intensidad que controló con éxito y en la que había aplicado, después del tiempo fuera, una corrección y había dado de nuevo la orden, que esta vez había sido obedecida. 
Sesión $4^{a}$ : En esta sesión los padres reconocieron que el comportamiento de la niña había mejorado, hacía cosas sola y seguía instrucciones, aunque tenían problemas a la hora de aplicar el tiempo fuera, debido a la larga duración e intensidad de las rabietas. Durante el tiempo de grabación, Elena tuvo una rabieta de alta intensidad y duración (33 minutos, aproximadamente) acompañada de agresiones y autolesiones.

Sesión $5^{a}$ : Los padres reconocieron que las rabietas eran menos frecuentes y de menor intensidad. Por primera vez en la sesión la madre aplicó un tiempo fuera contingente a una rabieta que duró unos 36 minutos. Esta rabieta no fue grabada porque apareció en un despacho desprovisto de equipo de grabación. Los padres describieron que hablaba más y con una mejor topografía vocal, y obedecía las órdenes que se sucedían durante el juego. Ambos padres jugaban con Elena en la casa durante 15 minutos todos los días. En esta sesión los padres pidieron ayuda sobre las negativas de Elena a dormir sola porque le daba miedo. Se les dijo que debían ser sistemáticos y consistentes al llevarla a su cama cada vez que se levantara. Entre la sesión $5^{\mathrm{a}}$ y la $6^{\mathrm{a}}$ se contactó telefónicamente con la madre. Ésta informó de un incremento de problemas después de la última sesión que habían sabido controlar. Los primeros 4 días se negó a dormir sola y lloró intensamente cada vez que la llevaban a su cama, pero a partir de ese día dormía sola con algunos llantos intermitentes que los padres ignoraban. La niña estaba más cariñosa con la madre. Los abuelos también reconocieron un cambio.

Sesión $6^{a}$ : Los padres aplicaban con más consistencia y de forma más sistemática las consecuencias al comportamiento de Elena, pero se observaron correcciones continuas por parte de ellos, lo que creaba un ambiente negativo entre los tres y dejaba poco espacio para el reforzamiento de conductas positivas. Se recomendó el incremento de esto último. En las rabietas de la niña aun aparecieron los insultos y agresiones. Llevaba 20 días durmiendo sola sin protestar y como premio, los padres de forma espontánea le compraron una muñeca. Durante la grabación de la interacción de Elena con sus padres por separado, apareció desobe- diencia y mutismo. Se observó que la relación entre padre e hija era distante y por esto los terapeutas insistieron que el padre jugara con ella unos 15 minutos diarios. Esta es la primera sesión en la que Elena no tuvo ninguna rabieta.

Sesión $7^{a}$ : Según los padres seguían teniendo problemas pero lo controlaban mejor en casa. Las dudas que plantearon se centraron en los fines de semana. Los padres estaban más seguros de cómo manejar estas conductas y se describieron así mismos como más sistemáticos y consistentes. Los terapeutas elogiaron estas mejoras y trabajaron en la eliminación de la tendencia que tenían de sermonear y de dar instrucciones innecesarias a la niña. Con el objetivo de mejorar la relación entre Elena y su padre, se acordó que cada vez que tuviera un día bueno, al acostarse su padre le leería un cuento, algo que le gustaba mucho. Durante la sesión apareció una rabieta con la terapeuta de 30 minutos, en la que Elena se hizo pipí. Durante la grabación, se observó que la niña seguía teniendo problemas para obedecer las órdenes. Los padres daban demasiadas instrucciones y su elogio se limitaba al muy bien, sin variar éste ni utilizar otras formas más naturales. La terapeuta presentó modelos a los padres, jugando con la niña y reforzándola de forma más natural.

Sesión 8a : Al comienzo de esta sesión y hubo una rabieta ante el terapeuta que aplicó un tiempo fuera. En esta sesión los padres parecían un poco desmotivados con el curso de la terapia, la madre describió el estado actual como estacionario. Reconocieron que se habían producido mejoras en la última semana, mostraba conductas positivas con más frecuencia, los llantos de las rabietas duraban menos y que había dormido todas las noches en su cama, sin reclamar atención. Aunque estaban preocupados por un incremento de conductas negativas. Los terapeutas lo interpretaron como una recuperación espontánea de la extinción. El trabajo en sesión se centró en una correcta aplicación de refuerzos sociales consecuentes a la conducta positiva. En estas grabaciones aparecieron seguimientos de órdenes, conductas de atención y conductas de generosidad con los terapeutas.

Sesión $9^{a}$ : Los padres describieron que la relación con el padre había mejorado, interac- 
tuando con más frecuencia y de manera más positiva. Durante la grabación, se pudieron observar múltiples conductas positivas (p.ej., hablar más con su padre, reaccionar sin quejas cuando perdía o se le retiraba un juego, aceptar un «no», etc.). Los padres fueron capaces de controlar situaciones que evocaban rabietas.

Sesión 10 ${ }^{a}$ : Esta sesión se realizó al mes y medio de la anterior con el objetivo de ir desvaneciendo el tratamiento. Los padres acudieron muy satisfechos. Durante los primeros 30 minutos de ésta, Elena se mantuvo sentada sin demandar la atención mientras sus padres hablaban. Elena había tenido solamente 3 rabietas en un mes y medio, con una duración de aproximadamente 15 minutos. El reforzamiento de los padres hacia las conductas positivas se había incrementado y lo aplicaban de manera natural. Las mejoras que reconocieron fueron: obedecer, jugar sola sin demandar atención de los padres, hablar más y con una mejor topografía vocal, pedir las cosas por favor y dar las gracias, relacionarse bien con otros niños, los conflictos con su hermano menor habían disminuido, estaba más contenta, daba mas besos a los demás, era más generosa, aceptaba perder, dormía sola y sin temores en su cama. Los padres reconocieron ser sensibles a sus conductas positivas y estar reforzando con naturalidad. Demoraban las demandas de Elena y atendían a éstas sólo cuando se realizan de manera correcta. Se realizó la grabación de la interacción de los dos padres a la vez con su hija, intercalando juegos con reglas y juegos más activos y desestructurados. En esta, Elena era obediente y generosa, aunque las instrucciones de los padres fueron menos directas y ocasionalmente surgieron conductas que se definen como desobedientes, que son corregidas por los padres y aceptadas por la niña.

Sesión 11 ${ }^{a}$ : Está sesión fue de seguimiento y se llevó a cabo a los tres meses de la anterior. En ella apareció una negativa que fue controlada por los padres. La madre enumeró múltiples conductas positivas de la niña durante este transcurso de tiempo, a la vez que el padre acariciaba a la niña de manera muy natural. Entre las mejoras que describieron: que la niña seguía órdenes sencillas, aceptaba el «no» por respuesta, guardaba turnos para hablar y respetaba cuando los demás están hablando sin interrumpir. Hablaba con más frecuencia y su vocabulario era más amplio y más claro. Estas conductas se generalizaron a otros contextos como en casa de los abuelos, en reuniones, en cenas con amigos, etc. Además, la niña aceptaba quedarse durante horas en casa de familiares sin los padres. Los padres intentaban que cada vez fuera más autónoma e independiente como: retirarle la barandilla de la cama, implicarla en el cuidado de su hermano menor, etc. Reconocieron una mejora en la relación entre los dos hermanos. Los padres comentaron haber tenido la necesidad de aplicar un tiempo fuera 3 ó 4 veces aproximadamente en estos 3 meses, que las rabietas han sido poco intensas y que durante éstas no había aparecido pipí. Los padres se sentían mejor y más seguros respecto a la relación con Elena. Los familiares y amigos reconocieron las mejoras. De acuerdo con los padres se decide dar el alta.

Se realizó un seguimiento telefónico a los 9 meses de la última sesión. La madre informó que Elena era obediente, dormía sin despertarse y sin llamadas de atención, mostraba su cariño a diario y le decía a su madre que la quería. La relación con su padre estaba muy mejorada. Era muy sociable, y hablaba con niños desconocidos. Utilizaba un vocabulario más amplio y hablaba de manera espontánea con más frecuencia. La relación con su hermano era buena. Aunque reconocieron que las negativas no se habían extinguido completamente. Las rabietas tenían una frecuencia de 1 cada 2-3 semanas pero de baja intensidad.

\section{RESULTADOS}

A lo largo de la intervención se ha modificado el comportamiento problemático de la niña reduciendo la intensidad, la duración y la frecuencia de las conductas problema. En la Tabla 1 , se pueden observar la frecuencia de conductas objetivo durante las grabaciones, la duración de éstas en minutos y el porcentaje de obediencia y desobediencia. Estos porcentajes se calcularon a través de la fórmula: [(frecuencia de obediencia)/(frecuencia de obediencia + fre- 
cuencia de desobediencia) $] \times 100$. Específicamente, escupir sólo aparece 9 veces en la primera sesión. Las autolesiones aparecen sólo en la sesión $4^{a}$ con una frecuencia de 29 . Las agresiones aparecen en las sesiones $1^{\mathrm{a}}$ y $4^{\mathrm{a}}$ con una frecuencia de 100 y 48, respectivamente. Las negativas van disminuyendo su frecuencia de 15 en la primera sesión hasta 0 en la $8^{\mathrm{a}}$ y $10^{\mathrm{a}}$, aunque se recupera en la $9^{\mathrm{a}}$ y $11^{\mathrm{a}}$. De la misma forma la desobediencia baja en frecuencia de 41 en la sesión $1^{\mathrm{a}}$ a 0 en la $9^{\mathrm{a}}$. Mostrando también una recuperación leve en las dos últimas. En cuanto a las rabietas, la conducta más problemática, aparecen en 6 de las 11 sesiones, aunque en la tabla aparecen sólo en 4, ya que las sesiones $3^{\mathrm{a}}$ y $5^{\mathrm{a}}$ no se grabaron. La frecuencia de la obediencia y su porcentaje se va incrementando conforme avanza el tratamiento. Por otro lado, en las grabaciones de las sesiones se puede observar que la mayoría de las conductas problema se llegan a eliminar (rabietas, agresiones, autolesiones, escupir) y se mantiene una frecuencia razonable en las restantes conductas (negativas y desobediencia).
En cuanto al porcentaje de obediencia y desobediencia se va incrementando y disminuyendo respectivamente, como se puede ver en la Figura 1. Consiguiendo un porcentaje de obediencia superior al $80 \%$ a partir de la sesión $6^{\mathrm{a}}$. $\mathrm{Y}$ al contrario en la misma sesión aparece una disminución inferior al 20\% en desobediencia que se mantiene. Por otro lado, la Figura 2 muestra la frecuencia de rabietas en el ambiente familiar durante el tratamiento. Según sus padres, la frecuencia aproximada pasa de unas 120 , es decir unas 4 rabietas diarias de promedio, a una frecuencia de 1 al mes en los tres meses del primer seguimiento (marzo, abril y mayo). Los meses de agosto y noviembre, diciembre y enero, los padres no llegaron a traer los registros y/o su frecuencia no era fiable. En el mes de julio hubo 19 rabietas, en septiembre 5 , en octubre hubo 16 pataletas y en febrero 2 . Sólo se han tenido en cuenta los datos fiables de la frecuencia de rabietas en casa. Los padres reconocen que hay una disminución de otros parámetros como la duración y la intensidad. Aunque en el seguimiento no se han consegui-

Tabla 1. Frecuencia de las conductas problema durante las grabaciones de las sesiones

\begin{tabular}{|c|c|c|c|c|c|c|c|c|c|}
\hline Sesiones & 1 & 2 & 4 & 6 & 7 & 8 & 9 & 10 & 11 \\
\hline Negativas & 15 & 2 & 6 & 5 & 1 & 0 & 8 & 0 & 4 \\
\hline Desobedecer & 41 & 32 & 37 & 6 & 15 & 1 & 0 & 4 & 2 \\
\hline Rabietas & 1 & 1 & 1 & 0 & 1 & 0 & 0 & 0 & 0 \\
\hline Agresión & 100 & 0 & 48 & 0 & 0 & 0 & 0 & 0 & 0 \\
\hline Autolesión & 0 & 0 & 29 & 0 & 0 & 0 & 0 & 0 & 0 \\
\hline Escupir & 9 & 0 & 0 & 0 & 0 & 0 & 0 & 0 & 0 \\
\hline Obedecer & 8 & 64 & 61 & 47 & 71 & 17 & 26 & 34 & 24 \\
\hline Duración de la grabación en minutos & 51 & 28 & 72 & 24 & 30 & 16 & 12 & 17 & 18 \\
\hline
\end{tabular}

Tabla 2. Frecuencia (\% entre paréntesis) de conductas a las que los padres prestan atención durante la grabación en cada sesión

\begin{tabular}{lccccccc}
\hline Sesiones & 1 & 4 & 6 & 7 & 9 & 10 & 11 \\
\hline Negativas & $4(20)$ & $1(2)$ & $2(7)$ & $0(0)$ & $0(0)$ & $0(0)$ & $0(0)$ \\
Desobedecer & $15(75)$ & $6(12)$ & $3(6)$ & $1(6)$ & $4(17)$ & $4(17)$ & $2(14)$ \\
Obedecer & $1(5)$ & $42(86)$ & $23(82)$ & $16(94)$ & $20(83)$ & $20(83)$ & $12(86)$ \\
Total & $20(100)$ & $49(100)$ & $28(100)$ & $17(100)$ & $24(100)$ & $24(100)$ & $14(100)$ \\
\hline $\begin{array}{l}\text { Duración de la interacción } \\
\text { en minutos }\end{array}$ & 51 & 72 & 11 & 20 & 12 & 17 & 18 \\
\hline
\end{tabular}




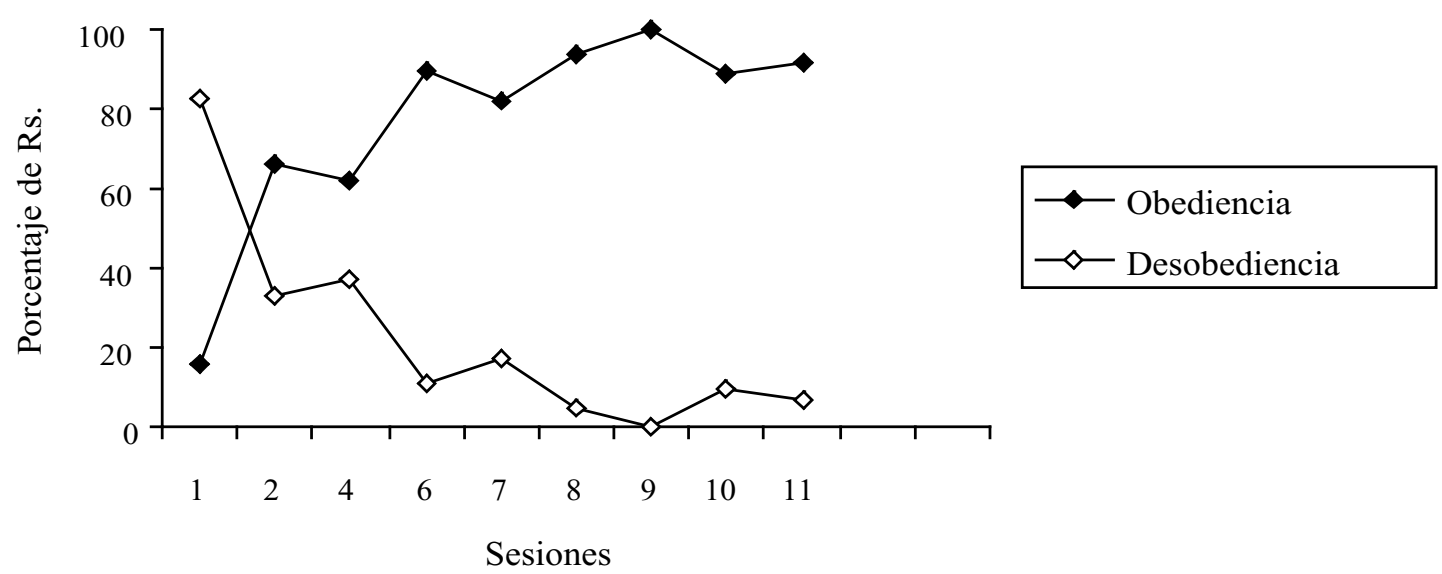

Figura 1. Porcentaje de obediencia y desobediencia durante las grabaciones de las sesiones

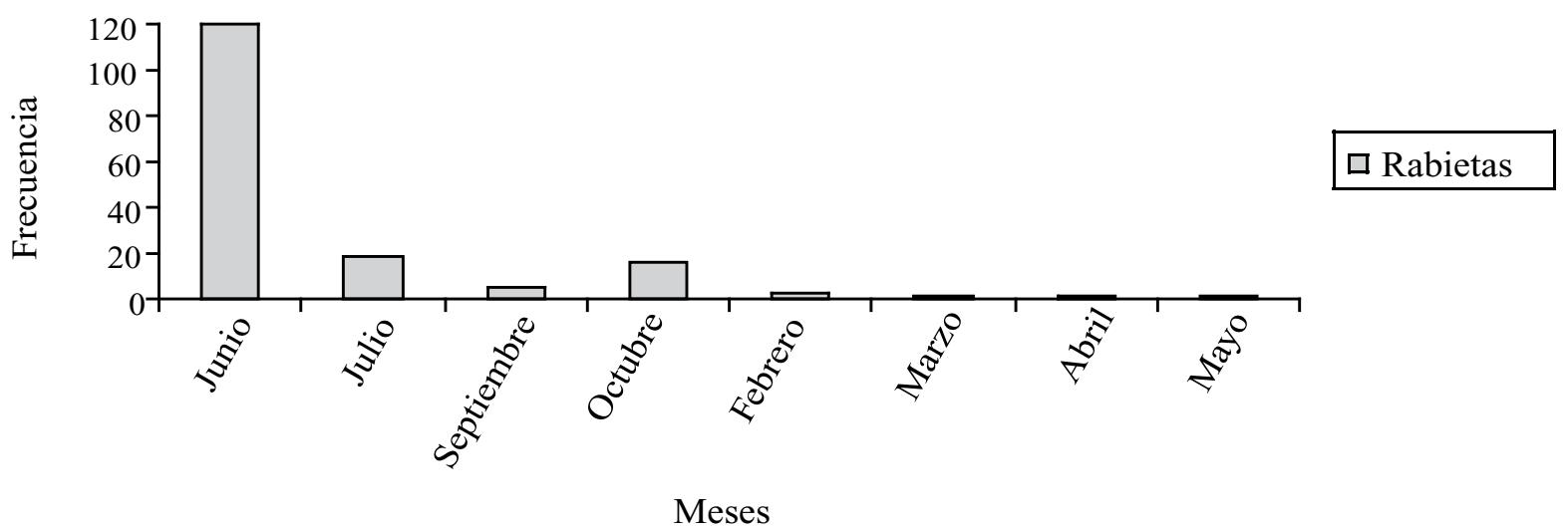

Figura 2. Frecuencia de rabietas por mes en casa durante el tratamiento

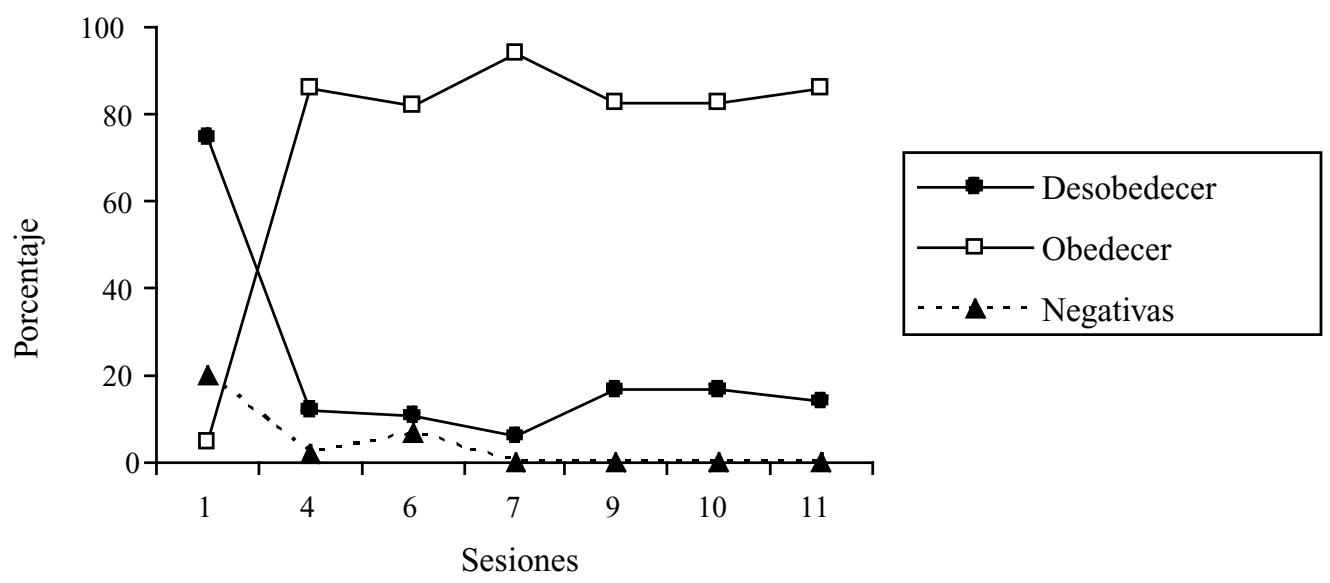

Figura 3. Porcentaje de conductas que van seguidas de la atención de los padres durante la grabación de las sesiones.

do eliminar las rabietas por completo, éstas fueron consideradas como normales. La duración de las rabietas en las sesiones clínicas varió de 36 minutos en la sesión $5^{\mathrm{a}}$ a 2 minutos en la $2^{\mathrm{a}}$ sesión.
En la Tabla 2 y en la Figura 3 se indica la frecuencia de conductas a las que los padres prestan atención durante las grabaciones de las sesiones. Los porcentajes se calcularon con la misma fórmula, esta vez usando en el divisor la 
sumatoria de negativas, obediencia y desobediencia. Para hacer estos cálculos sólo se tuvo en cuenta el periodo de tiempo en el que los padres interactuaron con la niña. En todas las sesiones intervenían por turnos ambos padres, en la sesión $9^{\mathrm{a}}$ intervino el padre solo. Como se puede observar, hay un cambio en la actitud de los padres a partir de la intervención a la hora de prestarle atención a las conductas de su hija que coincide con la disminución de las conductas problema de la niña y de los informes de análisis funcionales traídos a consulta durante la intervención.

A partir de la sesión $4^{\mathrm{a}}$ los padres reconocen algunas mejorías en el comportamiento de Elena. Pero es a partir de la sesión $5^{\text {a cuando los }}$ terapeutas aprecian un cambio más radical, tanto en los padres como en la niña. En la sesión $6^{\mathrm{a}}$ no aparece ninguna rabieta y en la $7^{\mathrm{a}}$ que la niña tuvo una rabieta en la que se orinó, se interpretó como una recuperación espontánea del proceso de extinción.

\section{DISCUSIÓN}

A la vista de los resultados podemos afirmar que la terapia de interacción padres-hijos ha resultado ser efectiva en el tratamiento de un caso de trastorno negativista desafiante. Hemos constatado un cambio significativo tanto del comportamiento de la niña como en el de los padres. También el análisis funcional de las conductas problema de Elena fue correcto. Las conductas problema estaban mantenidas por atención social de los propios padres y algunas (como las negativas) también por escape de algunas tareas. Los padres eran inconsistentes en sus consecuencias y asistemáticos. Gracias a esta intervención se consiguió que la atención fuera prestada a las conductas positivas y pro-sociales.

La PCIT ha demostrado ser efectiva en otros casos similares, y los datos que aquí presentamos vienen a reforzar esta conclusión. Se ha criticado que esta terapia no supone nada nuevo ya que se basa en métodos operantes. Sin embargo, su mayor aportación es el uso del juego como una manera natural de trabajar con niños pequeños y por lo tanto, promocionar la gene- ralización de los resultados. Es decir, usar la situación de juego como la mejor forma de trabajar con los niños y sus padres. Este trabajo confirma una vez más que uno de los factores que explican el TND son las prácticas de crianza, propuestas por Barkley et al. (1999), en el sentido de que los padres proporcionan poca atención o reforzamiento a las conductas adecuadas del niño.

El presente trabajo presenta algunas limitaciones. Metodológicamente se podrían haber realizado algunas mejoras en este estudio de caso en cuanto a la medición de las conductas. Por ejemplo, tomar medidas pre- y post- de la intervención con varios cuestionarios como por ejemplo el Inventario Eyberg de comportamientos en niños (García-Tornel, Eyberg, Calzada y Sáinz, 1998) y el Parenting Stress Index (Abidin, 1995). También, se podría haber calculado la fiabilidad con otro observador en el registro de las conductas durante las grabaciones. Aun así, nuestro estudio presenta datos bastante consistentes a favor de la efectividad de la terapia de interacción padres-hijos aplicada a un trastorno del comportamiento perturbador infantojuvenil.

\section{REFERENCIAS}

Abidin, R. R. (1995). Parenting stress index manual (3rd. ed.) Odessa, F.L. Psychological Assessment Resources.

American Psyquiatric Association (1994). DSM-IV. Manual diagnóstico y estadístico de los trastornos mentales. Barcelona: Masson.

Bagner, D. M. y Eyberg, S. M. (2007). Parent-child interaction therapy for disruptive behavior in children with mental retardation: A randomized controlled trial. Journal of Clinical Child and Adolescent Psychology, 36, 3, 418-429.

Barkley, R., Edwards, G.H., y Robin, A.L. (1999). Defiant teens: A clinician's manual for assessment and family intervention. New York: Guilford Publications.

Brinkmeyer M. y Eyberg S. M. (2003), Parent-child interaction therapy for oppositional children. En A.E. Kazdin y J.R. Weisz (Eds). Evidence-based psychotherapies for children and adolescents (pp. 204-223). New York: The Guilford Press.

Burke, J. D., Loeber, R. y Birmaher, B. (2002). Oppositional defiant and conduct disorder: A review of the past 10 years, part II. The Journal of the American Academy of Child and Adolescent Psychiatry, 41, 12751293. 
Cardo E, Meisel, G., Garcia-Banda, C., Palmer C., Riutort M., Bernad, M., y Servera, M. (2009) Trastorno negativista desafiante, aspectos relacionados con el sexo y el evaluador. Revista de Neurología, 48, 17-21.

Carrasco, M. A. y Del Barrio, M. V. (2007). El modelo de los cinco grandes como predictor de la conducta agresiva en población infanto-juvenil. Revista de Psicopatología y Psicología Clínica, 12, 1, 23-22.

Chaffin, M., Silovsky, J. F., Funderburk, B., Valle, L. A., Brestan, E. V., Balachova, T., Jackson, S., Lensgraf, J. y Bonner, B. L. (2004). Parent-child therapy with physsically abusive parents: efficacy for reducing future abuse reports. Journal of Consulting and Clinical Psychology, 72, 3, 500-510.

Connor, D.F. (2002). Aggression and antisocial behavior in children and adolescents: Research and treatment. New York: The Guilford Press.

Eyberg, S.M. (1988). Parent-child interaction therapy: Integration of traditional and behavioral concerns. Child and Family Behavior Therapy, 10, 33-46.

Eyberg, S.M. (1999). Parent-Child interation therapy (PCIT). Integrity checklists and session materials. University of Florida. Retrieved February 11, 2008, from the University of Florida PHHP website: http:// pcit.phhp.ufl.edu/

Eyberg, S. M., Funderburk, B. W., Hembree-Kigin, T. L., McNeil, C. B., Querido, J. G., y Hood, K. (2001). Parent-child interaction therapy with behavior problem children: One and two year maintenance of treatment effects in the family. Child \& Family Behavior Therapy, 23, 1-20.

Eyberg, S. M. y Matarazzo, R. G. (1980). Training parents as therapists: A comparison between individual parentchild interaction training and parent group didactic training. Journal of Clinical Psychology, 36, 492-499.

Ferro, R. (2006). La Psicoterapia analítica funcional: Estableciendo relaciones terapéuticas intensas y curativas. Psicología Conductual, 14, 3, 343-359.

Ferro, R., Valero, L. y López Bermúdez, M.A. (2007). Novedades y aportaciones desde la psicoterapia analítica funcional. Psicothema, 19, 452-458.
Ferro, R., Vives, M. C. y Ascanio, L. (2009). Novedades en el tratamiento conductual de niños y adolescentes. Clínica y Salud, 20, 2, 119-130.

García-Tornel, S., Eyberg, S., Calzada, E. y Sáinz, E. (1998). Trastornos del comportamiento en el niño: Utilidad del inventario Eyberg en la práctica diaria del pediatra. Pediatría Integral, 3, 3, 349-354.

Hembree-Kigin, T.L. y McNeil, C.B. (1995). Parent-child interaction therapy. New York: Plenum Press.

Herschell, A.D., Calzada, E. J., Eyberg, S.M., y McNeil, C.B. (2002). Parent-child interaction therapy: New directions in research. Cognitive and Behavioral Practice, 9, 9-16.

Hood, K. K. y Eyberg, S. M. (2003). Outcomes of parentchild interaction therapy: Mothers' reports of maintenance three to six years after treatment. Journal of Clinical Child and Adolescent Psychology, 32, 3, 419-429.

McNeil, C. B., Capage, L., Bahl, A. y Blanc, H. (1999). Important of early intervention for disruptive behavior problems: Comparison of treatment and waitlist-control groups. Early Education and Development, 10, 445454.

McNeil, C. B., Filcheck, H. A., Greco, L. A., Ware, L. M., y Bernard, R. S. (2001). Parent-child interaction therapy: Can a manualized treatment be functional? The Behavior Analyst Today, 2, 2, 106-114.

Portugal Fernández, A. y Arauxo Vilar, A. (2004). El modelo de Russell Barkley. Un modelo etiológico para comprender los trastornos de conducta. Revista de Psiquiatría y Psicología del Niño y del Adolescente, 4, 1, 54-64.

Rodríguez, M. A., Del Barrio, V. y Carrasco, M.A. (2009). Consistencia interparental y su relación con la agresión y la sintomatología depresiva en niños y adolescentes. Revista de Psicopatología y Psicología Clínica, 14, 1, 51-60.

Schuhmann, E. M., Foote, R. C., Eyberg, S. M., Boggs, S. R. y Algina, J. (1998). Efficacy of parent-child interaction therapy: Interim report of a randomized trial with short-term maintenance. Journal of Clinical Child Psychology, 27, 34-45. 